Service social

\title{
Implication économique et intégration sociale des femmes bénéficiaires d'une caisse d'épargne et de crédit au Sénégal
}

\section{Maïmouna Sourang, André Beaudoin et Élaine Carey-Bélanger}

Volume 47, numéro 3-4, 1998

Enfance : enjeux et réalités 2000

URI : https://id.erudit.org/iderudit/706803ar

DOI : https://doi.org/10.7202/706803ar

Aller au sommaire du numéro

Éditeur(s)

École de service social de l'Université Laval

ISSN

1708-1734 (numérique)

Découvrir la revue

Citer cet article

Sourang, M., Beaudoin, A. \& Carey-Bélanger, É. (1998). Implication économique et intégration sociale des femmes bénéficiaires d'une caisse d'épargne et de crédit au Sénégal. Service social, 47(3-4), 289-316.

https://doi.org/10.7202/706803ar
Résumé de l'article

L'objectif de cette étude est d'évaluer les effets du programme Caisse d'épargne et de crédit (CEC), à Thiès au Sénégal, sur le niveau d'implication économique et le degré d'intégration sociale des femmes bénéficiaires. Ces bénéficiaires, le groupe expérimental, sont comparées à un groupe témoin composé de femmes qui fréquentent le CPRS, mais ne bénéficient pas des services de ce programme. L'analyse des résultats montre que le programme CEC permet aux bénéficiaires d'avoir une implication économique et une intégration sociale plus forte par comparaison avec ungroupe de non-bénéficiaires. L'introduction des variables de contrôle fait ressortir que la scolarité a la même action déterminantechez les non-bénéficiaires que le programme CEC pour les bénéficiaires. On peut donc conclure que le programme est surtout efficace pour les femmes analphabètes ou peu scolarisées. 


\author{
Implication économique et \\ intégration sociale des femmes \\ bénéficiaires d'une caisse d'épargne \\ et de crédit au Sénégal
}

\author{
Maïmouna SoURANG \\ André BEAUDOIN \\ Élaine CAREY-BÉLANGER
}

L'objectif de cette étude est d'évaluer les effets du programme Caisse d'épargne et de crédit (CEC), à Thiès au Sénégal, sur le niveau d'implication économique et le degré d'intégration sociale des femmes bénéficiaires. Ces bénéficiaires, le groupe expérimental, sont comparées à un groupe témoin composé de femmes qui fréquentent le CPRS, mais ne bénéficient pas des services de ce programme. L'analyse des résultats montre que le programme CEC permet aux bénéficiaires d'avoir une implication économique et une intégration sociale plus forte par comparaison avec un groupe de non-bénéficiaires. L'introduction des variables de contrôle fait ressortir que la scolarité a la même action déterminante chez les non-bénéficiaires que le programme CEC pour les bénéficiaires. On peut donc conclure que le programme est surtout efficace pour les femmes analphabètes ou peu scolarisées.

This study proposes an evaluation of the effects of a programme that established a credit and savings fund at the Social Promotion and Reinsertion Centre in Sénégal, on the level of economic implication and social integration of the women who benefited from the programme. The beneficiaries, who represent the experimental group, are compared to a second group of women who attended and were active at the Centre but did not take part in this particular programme. The analysis of results indicates that the programme does enable the participants to have a stronger economic implication and social integration compared to the nonparticipating group. However, control variables show that scolarization has the same determining action as the programme for the non-beneficiaries. The programme seems to be most efficient for women with little formal schooling or who are illiterate. 
Cet article fait état d'une évaluation des effets d'un programme Caisse d'épargne et de crédit sur le niveau d'implication économique et le degré d'intégration sociale des femmes bénéficiaires. II pose la problématique de la marginalisation des femmes face au problème d'accès au crédit en milieu urbain et périurbain.

Depuis longtemps, les études ont fait ressortir que les approches résiduelles ne sont pas suffisantes pour satisfaire les besoins des populations défavorisées (Wilensky et Lebeaux, 1965). Ces constatations valent également pour le Sénégal en ce qui regarde le service social assistanciel hérité de l'administration française (Audibert, 1977). Dès les années 1980 d'autres actions furent jugées nécessaires pour atténuer l'impact des politiques d'ajustement structurel (PAS) ${ }^{1}$. La Direction de l'action sociale (DAS) au Sénégal s'est engagée dans une approche d'autopromotion, basée sur la participation et la valorisation du potentiel humain.

Le programme Caisse d'épargne et de crédit (CEC) fait partie de ce mouvement. II a été mis en œuvre par le Centre de promotion et de réinsertion sociale (CPRS) El Hadji Omar de Thiès, l'une des structures de base de la DAS. Le programme CEC est un moyen de soutenir la promotion socio-économique des femmes pauvres, qui représentent près des trois quarts de la population faisant appel au Centre. Même s'il existe plusieurs programmes pour les femmes pauvres, peu d'études permettent d'évaluer leurs effets et leur portée dans la perspective d'éclairer les décisions de planification qui s'imposent.

Notre étude tente de répondre à la question suivante : quels sont les effets du programme pour les femmes bénéficiaires? Plus précisément, nous voulons savoir quelles sont les retombées du programme pour ces bénéficiaires sur les plans économique et social, notamment au regard de leur équilibre extrafamilial. Deux questions retiennent plus particulièrement l'attention :

\footnotetext{
1 Les PAS sont des normes et des procédures imposées par les organismes de financement internationaux représentés par les institutions des accords de Bretton Woods que sont le Fonds monétaire international (FMI) et la Banque mondiale, pour aider les pays sous-développés à assainir leur économie nationale.
} 
1) Les bénéficiaires ont-elles des activités économiques plus développées que les non-bénéficiaires ? Sont-elles plus impliquées économiquement que les non-bénéficiaires?

2) Les bénéficiaires ont-elles un niveau d'intégration sociale plus élevé que celui des non-bénéficiaires?

L'objectif général de la recherche est de mesurer l'implication économique et le niveau d'intégration sociale des femmes bénéficiaires du programme. Les objectifs spécifiques visent à :

1) établir jusqu'à quel point les femmes bénéficiaires du programme se comparent aux femmes non bénéficiaires en ce qui concerne leur intégration au travail, leur formation et leur participation sociale;

2) cerner les changements produits par le programme par rapport à leur situation économique;

3) vérifier quels avantages elles tirent de cette situation économique;

4) voir ce qu'il en résulte sur le plan des considérations sociales.

Après une introduction au contexte géospatial et institutionnel du programme et une brève recension des écrits sur la question de l'implication économique et de l'intégration sociale des femmes en Afrique - et au Sénégal particulièrement - ainsi que sur la place qu'elles occupent dans le développement, nous définirons les concepts clés utilisés pour étudier l'implication économique et l'intégration sociale des femmes. Nous présenterons ensuite la méthodologie de l'étude et les caractéristiques sociodémographiques des femmes participant à l'étude. En dernier lieu, nous analyserons et discuterons les effets du programme sur l'implication économique et l'intégration sociale des bénéficiaires de la CEC.

\section{LE CONTEXTE GÉOSPATIAL ET INSTITUTIONNEL}

Rappelons que le Sénégal est un pays de l'Afrique de l'Ouest. II partage avec les pays de la zone sahélienne les mêmes problèmes liés à la crise économique. II a subi depuis 1979 quatre programmes successifs d'ajustement structurel. Les dépenses publiques pour l'éducation et les services sociaux ont diminué et la période d'austérité qui en a résulté a eu un effet négatif sur les 
groupes vulnérables, plus particulièrement les femmes et les enfants (Sow, 1995a).

Le Sénégal compte dix régions administratives, Thiès, la région de référence de l'étude, est la deuxième région après Dakar par sa population. La capitale régionale de cette région est la commune du même nom, Thiès. C'est une ville qui s'agrandit rapidement en raison d'un important flux migratoire dû à l'exode rural et du taux élevé de son accroissement démographique, 2,9\%. La ville a fortement ressenti la récession économique, plusieurs usines - sources d'emplois - ont disparu, accentuant le chômage et le sous-emploi (Kanouté, 1990). La marginalité et l'exclusion sociale prennent de l'ampleur, augmentant le nombre des demandeurs d'aide auprès des services sociaux.

Dans ce contexte, les femmes éprouvent des difficultés à subvenir aux besoins essentiels de la famille. Le CPRS, dont la mission est de prendre en charge les populations défavorisées, est sollicité par une nombreuse clientèle, dont 73 \% sont des femmes (CPRS, 1995).

C'est dans l'optique d'organiser ces femmes mères de familles démunies que le programme CEC a été créé pour faciliter l'accès des femmes, encadrées par le CPRS, au crédit et de leur donner des compétences dans la gestion de leurs activités génératrices de revenus. À court terme, le programme vise à appuyer les initiatives de création ou de développement d'activités de " petit commerce » au profit des bénéficiaires, c'est-à-dire des activités d'implication économique à moyen et à long terme, à accroître leur niveau de participation dans les réseaux sociaux pour atteindre l'intégration sociale et à parvenir à une décentralisation des caisses de crédit dans les quartiers défavorisés avec une autonomie de la gestion.

\section{LA LITTÉRATURE SUR LES FEMMES ET LE DÉVELOPPEMENT LES FEMMES DANS LE TIERS-MONDE, EN AFRIQUE FRANCOPHONE ET AU SÉNÉGAL}

À partir de la littérature consultée nous faisons le point sur l'état des connaissances en ce qui concerne la condition des femmes du tiers-monde dans le développement économique et social en général, leurs rôles et contributions. Ensuite, nous présentons les 
expériences économiques et le rôle de la femme dans la société sénégalaise.

\section{La place des femmes dans le développement économique et social}

\section{La situation des femmes dans le tiers-monde}

Bien que les femmes jouent un rôle capital dans l'agriculture et dans le développement économique en général, elles vivent un appauvrissement graduel et constant dans le tiers-monde. Cette situation d'appauvrissement est accentuée par la crise économique et par les effets pervers de la vision masculine dans les approches de développement (Boserup, 1983). En Afrique de l'Ouest, Savané met en cause la modernisation qui, selon elle, détruit les formes d'organisations paysannes par les cultures de rentes. Elle permet aux hommes d'accéder à l'économie financière au détriment des femmes (Savané, 1986). Ces constats sur la situation des femmes amènent à dire, dans un premier temps, que "le développement se faisait contre les femmes » (Tinker, 1979).

Cette sensibilisation sur la question des femmes a conduit à une révision des bases de l'aide internationale. Trois perspectives ont émergé à propos des femmes et du développement :

- L'intégration des femmes au développement (IFD): cette perspective s'appuie sur le paradigme de la modernisation et résume le problème des femmes à sa dimension économique.

- Femme et développement (FED) : cette approche a pour base la théorie de la dépendance. Elle insiste sur la relation entre la femme et le processus de développement et met en cause les structures inégalitaires et injustes des relations internationales.

- Genre et développement (GED) : il s'agit d'une perspective qui s'inspire de la théorie de la dépendance, de l'analyse de classe et de l'éducation populaire. Elle met l'accent sur les rôles sexuels, la construction sociale de production et reproduction à la base de l'oppression des femmes et prend en compte le rapport inégal entre les hommes et les femmes dans la participation à l'économie (Rathgeber, dans Dagenais et Piché, 1994). 
La dimension «femme » est au cœur de la réflexion sur le développement. Les chercheurs du tiers-monde proposent cependant une plus grande ouverture au genre et au développement par l'acceptation de la différence dans la pluralité (Voix $d u$ Sud, 1992, p. 122). Dans l'étude sur les maraîchères de Kinshasa, Gertrude Mianda parle de " contextualiser les rapports sociaux de sexe » pour permettre aux femmes d'émerger et d'exercer le pouvoir réel sur leur vie d'un point de vue pragmatique (Mianda, 1996). AFARD réclame la responsabilisation des femmes par des dispositions qui garantissent l'accès aux ressources précises, telles que la terre, le crédit, la formation, la technologie, l'éducation sociopolitique, ainsi qu'un espace politique pour participer dans les sphères publiques (AFARD, 1994).

\section{Rôles, contributions et expériences économiques}

Les femmes apportent une contribution appréciable et multiforme dans la société. Dixon-Mueller et Anker (1989), dans leur étude pour le Bureau international du travail (BIT), distinguent à ce propos cinq types de contributions économiques de la femme :

- la participation à la main-d'œuvre dans les différents secteurs de l'économie, mesurée par les taux d'activités économiques;

- l'utilisation du temps, mesurée par les heures passées à une variété d'activités économiques productives;

- la productivité du travail, définie par le volume de la production par unité de temps investie;

- la rétribution du travail avec les aspects économiques et sociaux, le temps de travail rémunéré ou non;

- la contribution au revenu du ménage.

On assiste aussi à l'élargissement de la responsabilité des femmes. Le tiers des ménages dans le monde est dirigé par des femmes; entre 15 et $25 \%$ en Afrique subsaharienne et $16 \%$ au Sénégal. Au Sénégal, ces femmes assument toutes les charges en l'absence des hommes, surtout dans le nord du pays (exode et 
émigration des hommes à l'extérieur pour chercher du travail) ${ }^{2}$ (Sow, 1995b; EDS II, 1994). En milieu urbain, les femmes, en plus du poids de leurs responsabilités, doivent s'adapter à un nouveau modèle de production. Elles s'investissent beaucoup pour des gains dérisoires, travaillant souvent de 6 à 18 heures par jour. Cette contribution n'est ni valorisée, ni prise en compte, mais considérée comme inhérente à leur condition de femme.

Cette situation des femmes crée une invisibilité qui rend leurs besoins absents des plans de développement. Pour accéder aux ressources, les femmes conçoivent des stratégies. On assiste au Sénégal à un essor du mouvement associatif (Bélanger et Sourang, 1994), mais ces organisations ne reposent que sur les modestes moyens du milieu et ne permettent pas de dépasser le cadre étroit des activités informelles. "Au Sahel, les femmes n'ont pas accès au crédit parce qu'elles sont pauvres et elles sont pauvres parce qu'elles n'ont pas accès au crédit » (Camiré, dans Champagne, 1989). Ainsi, les femmes sont en quelque sorte prises dans un cercle vicieux.

La différence des réalités socio-économiques des milieux rural et urbain ne change rien aux préoccupations des femmes qui ont partout les mêmes rôles et charges. Elles doivent faire face à des difficultés semblables dans la recherche de moyens pour accéder aux revenus. Les expériences de promotion économique en direction des femmes sont nombreuses et variées. Cependant, peu de ces expériences prennent en compte la spécificité de la femme, qui se caractérise par l'interdépendance des problèmes économiques et sociaux, une participation diversifiée avec des dimensions temporelle, culturelle, sociale et financière ainsi qu'une imbrication de leurs rôles. L'expérience des femmes de Madras en Inde fait partie de ces quelques réussites. Elle regroupe 7000 membres autour d'une banque, laquelle finance des activités économiques, mais soutient également les autres aspects des rôles de la femme (cérémonies, offrandes, etc.) qui sont en interrelation (Rathgeber, dans Dagenais et Piché, 1994).

2 Par chef de famille, on entend la personne reconnue comme ayant le leadership dans les affaires de la famille, y compris dans les affaires économiques. 
L'utilisation du savoir-faire local permet d'assurer une pleine participation des femmes. La tontine est une illustration de l'adaptation du savoir-faire local à des fins de promotion économique. Ces tontines revêtent plusieurs formes selon les besoins et les événements: tontines de toits, de champs, de deuils (Henry et collab., 1991). Les tontines devenues banques de quartier sont à l'origine de la puissance économique des " nanas benz », qui sont de riches commerçantes très connues pour leur sens des affaires et qui règnent dans les grands marchés du textile au Ghana, au Cameroun, au Togo (Sow, 1995a). Cette volonté d'adapter le crédit aux besoins des femmes anime aussi la Grameenbank du Bangladesh qui, avec ses 200000 adhérentes, a réussi à implanter des réseaux économiques solides à la base. Celle-ci doit son succès à l'entière responsabilisation des utilisatrices qui ont plein pouvoir au sein des conseils d'administration (Hook, 1990).

\section{La condition de la femme dans la société sénégalaise}

La femme sénégalaise a vécu, à l'instar de ses sœurs du tiersmonde, une dégradation constante de son statut, liée à des facteurs structurels (culturel, économique, politique). La cause principale de cette dégradation est l'accumulation des retards dans les domaines de l'éducation et de la formation, ce qui s'avère être un handicap majeur à l'accès au travail, aux revenus et au pouvoir décisionnel. Face à l'ajustement structurel, les femmes sont particulièrement désavantagées en raison des contraintes juridiques, réglementaires, sociales et économiques propres à leur statut (CEA, 1994).

Malgré cela, l'esprit entrepreneurial des femmes est apparu, ces dernières années, comme l'une des solutions les plus efficaces à la crise économique. Certaines femmes ont investi des secteurs aussi réservés que le commerce de gros dans des domaines très protégés comme l'importation du textile, auparavant un monopole libanais au Sénégal. Cette amélioration de l'esprit d'entreprise des femmes est une réalité, même dans les nouveaux quartiers en formation situés dans les zones périurbaines où la marginalité économique et sociale est très forte (Sarr, 1991). Ce n'est pas le cas cependant de la vaste majorité des femmes et on ne sait pas beaucoup de choses actuellement sur les facteurs qui, dans des 
programmes, ont une influence sur l'implication économique et l'intégration sociale des femmes. Nous allons définir ces deux importants concepts qui forment le cadre de référence de notre étude.

\section{CADRE CONCEPTUEL DE L'ÉTUDE :}

L'IMPLICATION ÉCONOMIQUE ET L'INTÉGRATION SOCIALE

Les deux concepts clés que sont l'implication économique et l'intégration sociale forment le cadre de référence de l'évaluation.

\section{L’implication économique}

À quoi réfère le concept d'implication économique des femmes? « Implication » renvoie à la notion d'appartenance: s'impliquer signifie s'engager, se donner à fond et participer (Dictionnaire de sociologie, 1989). L'implication est une extension du concept de participation, qui sous-tend toute une théorie et un modèle de développement. "L'adhésion des populations se mesurait à leur participation aux différentes phases d'un projet » (Van Giffen, dans Aubin, 1991). D'autres notions plus complexes ont été introduites à partir du concept de participation, comme la " participation-acceptation » et la "participation-identification » des besoins et solutions (Champagne, 1989). La participation dépasse la simple adhésion à un processus, elle implique la capacité des acteurs à comprendre les stratégies mises au point et à contrôler la marche de l'organisation.

Plus précisément, le concept d'implication économique est défini comme l'aménagement des ressources, les modalités selon lesquelles un individu ou une société affecte des moyens limités à la satisfaction de besoins nombreux et illimités (Didier, 1984). « La participation économique est un ensemble d'activités qui concourent à la production, à l'échange et à la répartition des richesses dans la société moderne ou dans un espace géographique » (Grawitz, 1988).

L'implication économique, en référence aux programmes de Caisse d'épargne et de crédit (CEC), appelle une participation active. Elle devient ainsi une notion dynamique pour représenter les activités de vente ou de production génératrices de revenus, 
réalisées individuellement, en coopérative ou dans le cadre de projets collectifs. C'est aussi le système d'échanges, de répartition et d'épargne des revenus dans la famille et la société. La participation économique comprend également les actions menées en vue d'améliorer les conditions de production des femmes. Elle inclut les facteurs d'appui à ce développement (Grawitz, 1988). Dans la présente étude, l'implication économique est mesurée en fonction de l'exercice d'activité économique, de la durée d'existence de l'activité économique, du temps consacré à l'activité économique, du nombre de jours par semaine, du type d'activité (individuel, collectif), des bénéfices réalisés et de l'utilisation de ces bénéfices. L'implication économique devient ainsi un moyen pour les femmes d'accéder à un plus grand bien-être qui débouche sur une intégration sociale plus accentuée.

\section{L'intégration sociale}

Le concept d'intégration sociale fait référence à l'identification du sujet à la famille, au milieu et à l'environnement socioculturel. II renvoie également au sentiment d'appartenance. Chaque membre a une obligation de participation volontaire en échange de la reconnaissance d'un statut et de ses corollaires que sont le pouvoir, la responsabilité et la considération. Ces transactions sont régies par les normes de l'institution ou du système social. Les membres, par l'exercice de leurs rôles et leur degré de participation, s'investissent en créant une solidarité réciproque et un enrichissement mutuel (Birou, 1986).

L'intégration sociale, qui implique un sentiment d'identification à un groupe et une intériorisation des normes ainsi que des valeurs dominantes, renferme aussi l'idée d'unité et d'harmonie (Grawitz, 1988; Durkheim, dans Ferréol, 1991). C'est la participation autonome des individus et des groupes à la vie en société grâce à l'incorporation de modèles, de comportements, de normes et de valeurs; d'où l'importance pour la société de développer des mécanismes d'aide, d'appui, ainsi que de nouveaux modes d'organisation des services (Beaudoin, 1987).

L'intégration de la femme, en particulier dans le tiers-monde, dépasse la seule conception d'un lien avec le développement et l'implication économique. C'est un concept multidimensionnel qui 
englobe non seulement la réciprocité, mais la notion d'affranchissement, d'autonomie et d' "empowerment " par sa capacité à participer à la prise de décision. Cette élévation devient un moyen de corriger la marginalisation (Boserup et Libjencrantz, 1975).

L'acquisition de compétences devient une condition essentielle pour l'intégration sociale (Diop, 1993). La formation permet d'acquérir et de mettre en pratique les aptitudes nécessaires à une bonne participation. Elle constitue le meilleur moyen de faire progresser l'intégration sociale, surtout pour des personnes défavorisées (Nations Unies, 1987).

L'intégration, selon Dutrénit (1989), est l'occasion d'un consensus sur les normes, les voies et les moyens de la réussite sociale. Elle est en même temps synonyme de participation, conflictuelle ou non. Elle est considérée comme le résultat d'efforts pour respecter des normes permettant l'exercice de la liberté de chacun, un minimum de communication avec les autres et une indispensable activité économique.

Cette définition sert de cadre de référence à l'étude. Dans le modèle de Dutrénit, on trouve quatre types d'intégration sociale : culturelle, normative, communicative et fonctionnelle. Nous avons retenu pour la présente étude deux dimensions: culturelle et fonctionnelle. Soucieux de cibler très précisément l'implication économique et l'intégration sociale des femmes, nous considérons ces deux dimensions avec leurs différents domaines comme étant les plus pertinentes, parce qu'elles permettent de faire ressortir les références à l'exercice des rôles dans le milieu. En ce sens, il faut préciser que l'intégration sociale est ici mesurée uniquement sur le plan extrafamilial.

À partir de la conception de Dutrénit, les dimensions intégratives s'opérationnalisent comme suit : chaque dimension compte deux sous-dimensions, soit les contributions et les rétributions, et ce, dans six domaines d'application: trois pour les contributions et trois pour les rétributions. Les contributions comprennent le travail, la formation et la participation sociale. Les rétributions comprennent le revenu, les secours/avantages et la considération sociale. 


\section{MÉTHODOLOGIE}

L'échantillon de l'étude est composé de deux groupes : un groupe expérimental qui est constitué de 60 bénéficiaires choisies de manière aléatoire à partir des 120 que compte le programme CEC et un groupe de 60 non-bénéficiaires choisies aussi au hasard parmi les 500 usagers du CPRS : c'est le groupe contrôle à des fins de comparaison.

Les instruments se composent d'un questionnaire et du Descripteur d'intégration sociale (DIS) de Dutrénit. Le questionnaire avec questions ouvertes et fermées sert à la collecte d'information sur les caractéristiques sociodémographiques des bénéficiaires, sur leur degré d'implication économique et sur les effets induits sur le plan politique. Pour mesurer l'intégration culturelle et fonctionnelle, le DIS intégré au questionnaire comprend 36 items. Les items d'intégration culturelle et fonctionnelle comprennent chacun deux sousdimensions : contributions et rétributions dans six domaines (travail, formation, participation sociale, revenu, secours et avantages, dération sociale). Chaque domaine compte trois items (voir figure 1). II s'agit donc d'une échelle théorique de 36 items, cotée de 1 à 36 points (oui $=1$, non $=0$ ).

La majorité des sujets parlant le wolof et étant analphabètes, les instruments ont été traduits en wolof et appliqués par entrevue. On a analysé les résultats à l'aide de méthodes bivariées.

\section{LES CARACTÉRISTIQUES DES RÉPONDANTES}

L'âge des répondantes se situe entre 23 et 70 ans. Les 40 ans et moins sont majoritaires. Les non-bénéficiaires sont plus jeunes que les bénéficiaires. En général, les répondantes sont mariées et une forte proportion d'entre elles sont dans des ménages polygames. Le nombre d'enfants par répondante est égal à 6,7 - chiffre plus élevé que la moyenne nationale, qui est de 6,0. Les répondantes sont très majoritairement analphabètes, plus des deux tiers ne sachant ni lire ni écrire. Les bénéficiaires sont moins scolarisées que les femmes du groupe de comparaison. Par contre, la formation en gestion concerne sept bénéficiaires toutes membres du comité de gestion du programme CEC. La durée de fréquentation du CPRS s'échelonne entre un et 23 ans, les 
bénéficiaires ayant cependant une plus longue fréquentation du CPRS. Les répondantes ont un même niveau de participation aux différents autres types d'activités du CPRS.

Figure 1 : Descripteur d'intégration sociale

\begin{tabular}{|c|c|c|}
\hline & Dimension culturelle & Dimension fonctionnelle \\
\hline \multicolumn{3}{|l|}{ Contributions } \\
\hline Domaine travail & $\begin{array}{l}\text { 1. Avoir donné son avis } \\
\text { sur une ou des activités } \\
\text { de travail extrafamiliales } \\
\text { dans les six derniers } \\
\text { mois. } \\
\text { 2. Avoir l'impression de } \\
\text { réussir une bonne } \\
\text { gestion de son activité } \\
\text { de travail. } \\
\text { 3. Avoir organisé son } \\
\text { activité économique } \\
\text { de façon à économiser } \\
\text { du temps et de l'argent. }\end{array}$ & $\begin{array}{l}\text { 19. Avoir raté une occasion } \\
\text { qui aurait pu profiter à } \\
\text { son activité de travail. } \\
\text { 20. Avoir un rythme de travail } \\
\text { supérieur à celui de } \\
\text { l'année dernière à la } \\
\text { même période. } \\
\text { 21. Avoir participé à la } \\
\text { fondation d'une activité } \\
\text { ou action économique } \\
\text { avec d'autres personnes } \\
\text { en dehors de son activité } \\
\text { principale actuelle. }\end{array}$ \\
\hline $\begin{array}{l}\text { Domaine } \\
\text { formation }\end{array}$ & $\begin{array}{l}\text { 4. Avoir rencontré une } \\
\text { autorité dans le but } \\
\text { d'améliorer son activité } \\
\text { de travail. } \\
\text { 5. Avoir eu l'occasion de } \\
\text { transmettre ou d'expli- } \\
\text { quer à d'autres femmes } \\
\text { les informations reçues } \\
\text { dans le cadre des } \\
\text { programmes au Centre. } \\
\text { 6. Avoir reçu une formation } \\
\text { dans un autre domaine } \\
\text { au cours des deux } \\
\text { dernières années. }\end{array}$ & $\begin{array}{l}\text { 22. Avoir fait des démarches } \\
\text { pour obtenir des } \\
\text { informations pouvant } \\
\text { résoudre un problème de } \\
\text { travail. } \\
\text { 23. Avoir participé à une } \\
\text { session de formation ou } \\
\text { réunion qui a été utile } \\
\text { dans une tâche précise } \\
\text { de travail. }\end{array}$ \\
\hline $\begin{array}{l}\text { Domaine } \\
\text { participation } \\
\text { sociale }\end{array}$ & $\begin{array}{l}\text { 7. Être membre d'une } \\
\text { association d'entraide. } \\
\text { 8. À l'intérieur de } \\
\text { l'association, avoir } \\
\text { occupé un poste de } \\
\text { responsabilité. } \\
\text { 9. Connaître une organi- } \\
\text { sation économique } \\
\text { extérieure utile à ses } \\
\text { activités. }\end{array}$ & $\begin{array}{l}\text { 25. Avoir une carte d'identité } \\
\text { valide. } \\
\text { 26. S'être adressée au moins } \\
\text { une fois au CPRS dans } \\
\text { les six derniers mois. } \\
\text { 27. Avoir aidé une autre } \\
\text { femme du milieu à ré- } \\
\text { soudre un problème. }\end{array}$ \\
\hline
\end{tabular}




\begin{tabular}{|c|c|c|}
\hline & Dimension culturelle & Dimension fonctionnelle \\
\hline \multicolumn{3}{|l|}{ Rétributions } \\
\hline $\begin{array}{l}\text { Domaine } \\
\text { revenus }\end{array}$ & $\begin{array}{l}\text { 10. Avoir été complimentée } \\
\text { pour le choix et la tenue } \\
\text { de ses vêtements. } \\
\text { 11. Avoir suivi le journal } \\
\text { radiodiffusé ou télévisé } \\
\text { au moins une fois dans } \\
\text { la semaine. } \\
\text { 12. Avoir participé à plus } \\
\text { de deux cérémonies } \\
\text { dans les deux derniers } \\
\text { mois. }\end{array}$ & $\begin{array}{l}\text { 28. Qualifier sa situation } \\
\text { économique de cette } \\
\text { année de meilleure à } \\
\text { celle de l'année dernière. } \\
\text { 29. Avoir amélioré son cadre } \\
\text { de vie au cours de cette } \\
\text { année. } \\
\text { 30. Connaître d'autres } \\
\text { moyens pour augmenter } \\
\text { ses revenus. }\end{array}$ \\
\hline $\begin{array}{l}\text { Domaine } \\
\text { secours et } \\
\text { avantages }\end{array}$ & $\begin{array}{l}\text { 13. Avoir reçu une aide } \\
\text { morale d'une autre } \\
\text { personne hors de la } \\
\text { famille pour une diffi- } \\
\text { culté liée à son activité } \\
\text { dans les trois derniers } \\
\text { mois. } \\
\text { 14. Avoir été conseillée par } \\
\text { un membre du } \\
\text { personnel du centre ou } \\
\text { un professionnel sur } \\
\text { son activité de travail. } \\
\text { 15. Avoir bénéficié d'un } \\
\text { soutien technique dans } \\
\text { son milieu. }\end{array}$ & $\begin{array}{l}\text { 31. Entretenir de bons } \\
\text { rapports avec les } \\
\text { personnes avec les- } \\
\text { quelles elle est reliée par } \\
\text { son activité autres que la } \\
\text { clientèle. } \\
\text { 32. Avoir participé à des } \\
\text { actions récréatives de } \\
\text { groupe dans les deux } \\
\text { derniers mois. } \\
\text { 33. Avoir pu acquérir cette } \\
\text { année plus de bijoux, } \\
\text { meubles, autres articles } \\
\text { du même genre que } \\
\text { l'année précédente. }\end{array}$ \\
\hline $\begin{array}{l}\text { Domaine } \\
\text { considération } \\
\text { sociale }\end{array}$ & $\begin{array}{l}\text { 16. Avoir été félicitée pour } \\
\text { une idée ou une ini- } \\
\text { tiative prise durant } \\
\text { l'année écoulée dans le } \\
\text { cadre d'une activité. } \\
\text { 17. Avoir échangé des } \\
\text { idées ou expériences } \\
\text { avec d'autres femmes } \\
\text { dans le cadre de leurs } \\
\text { activités économiques. } \\
\text { 18. Avoir été élue à un } \\
\text { poste de responsabilité } \\
\text { dans un mouvement de } \\
\text { promotion féminine. }\end{array}$ & 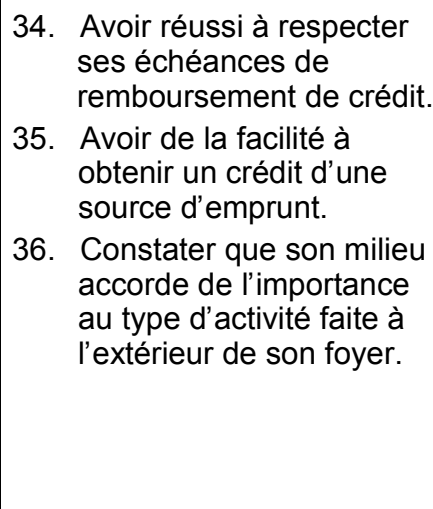 \\
\hline
\end{tabular}


Les répondantes du programme ont un profil analogue à celui du groupe témoin, sauf pour l'âge, la scolarité et la durée de fréquentation $(p<0,05)$. Ces dernières variables seront utilisées comme variables de contrôle à des fins de comparaison ultérieure.

\section{Les différences entre les bénéficiaires du programme CEC et les autres quant à leur l'implication économique}

L'hypothèse vérifiée par l'étude est que les bénéficiaires du programme CEC s'impliqueront plus sur le plan économique que ne le feront les non-bénéficiaires. Si l'hypothèse se confirme, il deviendrait possible d'attribuer au programme une certaine influence, tout en étant conscient que la relation de causalité n'est pas démontrée directement. Le tableau 1 fait une synthèse des variables indiquant une différence significative $(p<0,05)$ entre les bénéficiaires et les non-bénéficiaires pour ce qui est de l'implication économique.

Tableau 1

Synthèse des variables liées à l'implication économique

\begin{tabular}{|c|c|c|c|c|c|c|}
\hline \multirow[t]{2}{*}{$\begin{array}{l}\text { Type d'implication } \\
\text { économique }\end{array}$} & \multicolumn{2}{|c|}{$\begin{array}{c}\text { Non-bénéficiaires } \\
(\mathrm{n}=60)\end{array}$} & \multicolumn{2}{|c|}{$\begin{array}{l}\text { Bénéficiaires } \\
(\mathrm{n}=60)\end{array}$} & \multicolumn{2}{|c|}{$\begin{array}{c}\text { Ensemble } \\
(\mathrm{N}=120)\end{array}$} \\
\hline & $n$ & $\%$ & $\mathrm{n}$ & $\%$ & $\mathbf{N}$ & $\%$ \\
\hline $\begin{array}{l}\text { Exercice d'activité } \\
\text { économique }^{1}\end{array}$ & 35 & 58,4 & 59 & 98,2 & 94 & 78,3 \\
\hline Obtention crédit argent ${ }^{2}$ & 22 & 23,3 & 59 & 62,9 & 81 & 86,2 \\
\hline $\begin{array}{l}\text { Plus de } 2 \text { ans de durée } \\
\text { d'exercice }\end{array}$ & 18 & 30,0 & 58 & 96,8 & 73 & 63,4 \\
\hline Bénéfices ${ }^{4}$ & 27 & 45,0 & 53 & 88,0 & 80 & 75,0 \\
\hline Utilisation des bénéfices & & & & & & \\
\hline Épargne ${ }^{5}$ & 7 & 12,0 & 27 & 45,0 & 34 & 28,3 \\
\hline Investissement $^{6}$ & 21 & 35,0 & 53 & 88,0 & 74 & 61,7 \\
\hline
\end{tabular}

1. Khi carré $=4,386 ; d l=1 ; p=0,01$ ( $p<0,05$ significatif $)$.

2. Khi carré $=8,612 ; d l=2 ; p=0,000$ ( $p<0,05$ significatif).

3. Khi carré $=6,910 ; d l=2 ; p=0,001$ ( $p<0,05$ significatif $)$.

4. Khi carré $=10,410 ; d l=1 ; p=0,03$ ( $p<0,05$ significatif).

5. Khi carré $=5,312 ; d l=1 ; p=0,01$ ( $p<0,05$ significatif $)$.

6 . Khi carré $=6,002 ; d l=1 ; p=0,000$ ( $p<0,05$ significatif).

II ressort de ce tableau que les bénéficiaires se distinguent des non-bénéficiaires sur les aspects suivants. Ainsi : 
1) Les bénéficiaires exercent plus d'activités économiques. La présence du programme CEC qui accorde des prêts réguliers aux bénéficiaires suivant un système rotatif, comme l'indiquent les données sur leur accès au crédit, pourrait expliquer cet état de choses. La CEC est une source de financement qui accorde des prêts à des conditions avantageuses, par rapport au marché privé, et protège les bénéficiaires des abus de l'usure. Les autres sources de financement - crédit fournisseur, épargne personnelle et don - qui pourraient agir comme facteurs d'influence n'ont pas de lien avec le programme CEC $(p>0,05)$.

2) Les bénéficiaires exercent leurs activités économiques depuis une plus longue période, elles ont une continuité plus marquée dans l'exercice de l'activité économique, ce qui paraît être un indicateur de stabilité. Les répondantes en général travaillent plus à titre individuel ou en famille qu'à l'intérieur d'initiatives collectives. Le bas niveau d'éducation et de formation en général (scolarisation, alphabétisation, gestion) pourrait expliquer le nombre réduit de projets collectifs qui nécessitent une organisation plus poussée.

3) Les bénéficiaires sont plus nombreuses à réaliser des bénéfices, elles épargnent davantage et réinvestissent dans leurs activités économiques. II n'en reste pas moins qu'une proportion importante des bénéfices est utilisée pour la consommation, tant pour les bénéficiaires que pour les non-bénéficiaires. Rappelons que les répondantes sont toutes des mères issues de familles démunies. Elles viennent solliciter les services du CPRS pour améliorer leurs conditions de vie. II est donc normal que les retombées des activités économiques soient en partie utilisées pour améliorer la qualité de vie.

En somme, les bénéficiaires du CEC affichent un niveau d'implication économique plus élevé que celui des non-bénéficiaires. Ces résultats sont confirmés par la plupart des variables de contrôle : ni l'âge ni la durée de fréquentation du CPRS $(p>0,05)$ ne sont en cause. II est donc clair que c'est la participation à la CEC qui les distingue des autres. 
La scolarité introduit toutefois une nuance : les non-bénéficiaires scolarisées ont une implication économique aussi forte que les bénéficiaires, contrairement aux non-bénéficiaires qui ne sont pas scolarisées. L'interprétation quant aux effets du programme CEC mérite aussi d'être nuancée. Les bénéficiaires moins scolarisées réussissent avec la CEC, tandis qu'un plus grand nombre de nonbénéficiaires non scolarisées n'ont pas d'activité économique. Par contre, les non-bénéficiaires scolarisées ont tendance à mieux se débrouiller par elles-mêmes. Cela montre que le programme CEC est utile et plus adapté aux femmes analphabètes.

\section{L'INTÉGRATION SOCIALE DES BÉNÉFICIAIRES DU PROGRAMME CEC}

L'implication économique des bénéficiaires à travers les diverses activités qu'elles mènent engendre l'accroissement de leurs compétences dans les domaines investis. Cette implication se fait dans une dynamique d'autopromotion qui rend plus visible le rôle de la femme par rapport aux autres acteurs de l'environnement extrafamilial. On devrait en conséquence se trouver en présence d'une plus forte intégration sociale.

Comme nous l'avons indiqué précédemment, nous avons opté pour l'utilisation de deux dimensions du Descripteur d'intégration sociale (DIS) de Dutrénit, que nous avons adapté pour comparer l'intégration sociale des bénéficiaires avec celle des nonbénéficiaires : les dimensions culturelle et fonctionnelle. L'analyse est faite pour les quatre sous-échelles, soit deux sous-échelles pour chaque dimension : les contributions et les rétributions.

Toutefois, avant d'effectuer les comparaisons entre les deux sousgroupes, il était nécessaire de s'assurer de la consistance interne du DIS. À cette fin, les coefficients « alpha » de Crombach ont été calculés pour chaque sous-échelle. Les valeurs obtenues pour l'alpha de Crombach varient de 0,73 à 0,78 , sauf pour la souséchelle des rétributions de la dimension fonctionnelle. Pour cette sous-échelle, l'alpha initial était de 0,43 . Après le retrait des items $\mathrm{n}^{\mathrm{os}} 33,35,36$, la valeur obtenue pour l'échelle réduite à six items est de 0,53 . Nous avons décidé de garder cette sous-échelle malgré cet alpha plutôt faible, en mettant des réserves sur les résultats, ce qui constitue une limite pour l'étude. L'analyse est donc

conduite sur la base des trois premières sous-échelles de neuf 
items cotées de 0 à 9 points, soit les sous-échelles contribution fonctionnelle, rétribution culturelle et contribution culturelle, et de la sous-échelle rétribution fonctionnelle de six items, cotée de 0 à 6 points. Le tableau 2 présente les résultats de cette analyse.

\section{Tableau 2}

\section{Synthèse des résultats du DIS}

\begin{tabular}{|l|c|c|c|c|}
\hline \multirow{2}{*}{ Les sous-échelles* } & \multicolumn{2}{|c|}{$\begin{array}{c}\text { Non-bénéficiaires } \\
(\mathrm{N}=60)\end{array}$} & \multicolumn{2}{c|}{$\begin{array}{c}\text { Bénéficiaires } \\
(\mathrm{N}=60)\end{array}$} \\
\cline { 2 - 5 } & Moyenne & Écart type & Moyenne & Écart type \\
\hline Contribution culturelle & 3,32 & 2,28 & 5,85 & 1,49 \\
Rétribution culturelle & 4,08 & 1,70 & 5,46 & 1,65 \\
Contribution fonctionnelle & 3,91 & 2,16 & 6,91 & 1,89 \\
Rétribution fonctionnelle & 2,65 & 1,68 & 3,80 & 1,52 \\
\hline CONTRIBUTIONS & 7,40 & 3,68 & 11,31 & 2,70 \\
RÉTRIBUTIONS & 7,65 & 4,24 & 13,51 & 2,25 \\
\hline
\end{tabular}

* Test $t$ de Student significatif $p<0,05$.

\section{LA DIMENSION CULTURELLE DE L'INTÉGRATION}

L'intégration culturelle est la relation entre les traits qui constituent des normes culturelles d'un milieu au sens où ils acquièrent l'adhésion. Elle varie selon la concordance ou la non-concordance et renseigne dans le cadre de l'étude sur l'état d'harmonie des répondantes avec leur environnement social. Les contributions représentent toutes les formes de participation et les rétributions sont les gains qui résultent de cette participation.

Aux deux sous-échelles de la dimension culturelle, les bénéficiaires se répartissent autour de valeurs moyennes significativement plus élevées, tandis que les non-bénéficiaires ont des valeurs plus faibles. Ces résultats mettent en évidence que les bénéficiaires ont un niveau d'intégration sociale plus élevé que les nonbénéficiaires dans la dimension culturelle $(p<0,05)$. Les bénéficiaires qui s'investissent par l'activité économique apportent une contribution plus forte au développement de leur milieu sur le plan culturel. En même temps, cette participation sociale est accompagnée de rétributions sous la forme d'un état d'harmonie avec l'environnement social, cadre propice à l'épanouissement. On peut donc estimer que le programme permet aux bénéficiaires 
d'avoir une intégration culturelle plus forte que celle des nonbénéficiaires.

\section{LA DIMENSION FONCTIONNELLE DE L'INTÉGRATION}

L'intégration fonctionnelle mesure le degré d'interdépendance entre les échanges de services et la division du travail. Elle renseigne sur les capacités de création de liens des répondantes avec les éléments essentiels du milieu pour la réalisation des activités génératrices de revenus. Les résultats dans la dimension fonctionnelle confirment la supériorité des bénéficiaires sur les non-bénéficiaires. Les bénéficiaires sont plus aptes à créer des liens dans le milieu pour la réalisation de leurs activités économiques. Elles sont en plus forte interdépendance avec les services reliés à leur travail. Elles ont une plus forte intégration dans le réseau économique et réussissent mieux leur travail.

En résumé, les résultats du DIS à quatre sous-échelles ont démontré une différence significative de niveau d'intégration sociale entre les deux catégories de répondantes. Les participantes à la CEC appelées bénéficiaires ont un niveau d'intégration sociale plus élevé dans les dimensions culturelle et fonctionnelle.

\section{LES CONTRIBUTIONS ET LES RÉTRIBUTIONS ET LEURS EFFETS INDUITS}

De façon synthétique, comme l'indique la dernière partie du tableau 2, les bénéficiaires du CEC ont des contributions et des rétributions plus élevées que les non-bénéficiaires $(p>0,05)$. Les femmes bénéficiaires contribuent davantage par l'activité économique. Les habiletés et compétences acquises dans le travail augmentent leur expertise dans le milieu. Ces femmes sont mieux rétribuées par leur intégration dans le circuit économique, ce qui reflète une acceptation dans l'environnement. Les revenus générés permettent d'avoir des transactions positives dans le contexte de monétarisation des milieux urbains. Cette situation d'équilibre avec l'environnement fournit un espace de liberté pour négocier une place plus importante dans la société. Enfin, les revenus donnent aux femmes plus de pouvoir sur leur vie, plus de capacités d'agir ou d'empowerment (Dumouchel et Thède, 1986). 
Mais est-ce que les femmes exercent ce pouvoir? Bien que nous n'ayons pas fait de recherche directe dans ce sens, nous avons tenté d'apprécier jusqu'à quel point l'accès au crédit en vue d'un investissement générateur de revenus peut engendrer une participation accrue dans la vie politique et faire naître le besoin d'exercice du rôle de leadership dans ce domaine. Les résultats montrent que le programme ne donne pas aux bénéficiaires des compétences pour une participation politique. Ces résultats confirment les conclusions d'une autre étude sur les femmes au Sénégal : les femmes n'exercent pas leurs droits politiques (Silva et Juteau, 1993).

On sait par ailleurs que dans les mouvements associatifs l'optique première des femmes est de régler des problèmes économiques et non d'affirmation ou de quête de pouvoir. La mutation vers les activités politiques procède souvent de facteurs exogènes au groupe. Les femmes conscientes de leur poids utilisent ces rapports pour renforcer leur pouvoir économique. C'est seulement après qu'elles se lancent dans la recherche d'un statut de leadership politique et de décideur.

Ces résultats confirment la supériorité des bénéficiaires du programme CEC sur les non-bénéficiaires dans les deux sous-échelles de contributions et de rétributions. Les bénéficiaires ont un niveau d'intégration sociale plus élevé que les non-bénéficiaires. Toutefois, cette intégration ne déborde pas sur un engagement politique.

\section{L'INFLUENCE DE L'ÂGE ET DE L’ÉDUCATION}

Néanmoins, ainsi qu'il a été constaté au départ de l'étude, les bénéficiaires se distinguaient des non-bénéficiaires en ce qui concerne l'âge, la scolarité et la durée de fréquentation du programme. Pour l'analyse finale, nous n'avons retenu que deux variables de contrôle sur les trois initialement prévues, soit l'âge et la scolarité. En effet, l'âge et la durée de fréquentation se confondent, étant donné que la durée de fréquentation des plus âgées est plus longue que celle des plus jeunes. En conséquence, de ces deux variables seul l'âge est retenu. Le tableau 3 présente les résultats de cette analyse. 
Tableau 3

Influence de l'âge et de la scolarité sur la différence entre les contributions et les rétributions des bénéficiaires et des non-bénéficiaires

\begin{tabular}{|c|c|c|c|c|}
\hline & \multicolumn{2}{|c|}{ Contributions } & \multicolumn{2}{|c|}{ Rétributions } \\
\hline & Bénéficiaires & $\begin{array}{c}\text { Non- } \\
\text { bénéficiaires }\end{array}$ & Bénéficiaires & $\begin{array}{c}\text { Non- } \\
\text { bénéficiaires }\end{array}$ \\
\hline \multicolumn{5}{|l|}{$\begin{array}{l}\text { Âge : } \\
-40 \text { ans et moins }\end{array}$} \\
\hline - 40 ans et moins & & & & \\
\hline Nombre & 28 & 39 & 28 & 39 \\
\hline Moyenne & 11,47 & 7,42 & 13,01 & 7,91 \\
\hline Écart type & 2,50 & 3,11 & 2,78 & 3,02 \\
\hline Valeurs $t$ et $p$ & \multicolumn{2}{|c|}{8,$42 ; p<0,05$} & \multicolumn{2}{|c|}{9,$42 ; p<0,05$} \\
\hline - Plus de 40 ans & & & & \\
\hline Nombre & 32 & 21 & 32 & 21 \\
\hline Moyenne & 11,31 & 7,81 & 13,60 & 7,99 \\
\hline Écart type & 2,61 & 3,78 & 2,19 & 4,00 \\
\hline Valeurs $t$ et $p$ & \multicolumn{2}{|c|}{8,$50 ; p<0,05$} & \multicolumn{2}{|c|}{10,$48 ; p<0,05$} \\
\hline \multicolumn{5}{|l|}{$\begin{array}{l}\text { Scolarité : } \\
\text { - Non scolarisées }\end{array}$} \\
\hline Nombre & 54 & 36 & 54 & 36 \\
\hline Moyenne & 10,99 & 5,91 & 13,40 & 6,71 \\
\hline Écart type & 2,85 & 4,31 & 2,29 & 3,99 \\
\hline Valeurs $t$ et $p$ & \multicolumn{2}{|c|}{8,$71 ; p<0,05$} & \multicolumn{2}{|c|}{7,$42 ; p<0,05$} \\
\hline - Scolarisées & & & & \\
\hline Nombre & 6 & 24 & 6 & 24 \\
\hline Moyenne & 11,08 & 10,90 & 13,42 & 11,92 \\
\hline Écart type & 2,70 & 2,32 & 2,30 & 2,95 \\
\hline Valeurs $t$ et $p$ & \multicolumn{2}{|c|}{0,$84 ; p>0,05$} & \multicolumn{2}{|c|}{0,$95 ; p>0,05$} \\
\hline
\end{tabular}

La comparaison faite entre les 40 ans et moins et les plus de 40 ans ne change pas les constatations initiales de différences entre les non-bénéficiaires et les bénéficiaires en ce qui concerne les contributions et les rétributions $(p>0,05)$. La différence est donc attribuable au programme plutôt qu'à l'âge.

Pour ce qui est de la scolarité, on constate tant dans les contributions que dans les rétributions une différence significative, chez les non-scolarisées, entre les bénéficiaires et les non-bénéficiaires 
$(p<0,05)$. II est donc possible d'affirmer que pour les femmes non scolarisées c'est le programme CEC qui fait toute la différence. Pour les scolarisées, par contre, cette différence n'existe pas $(p>0,05)$ entre les bénéficiaires et les non-bénéficiaires. II semblerait par conséquent que le niveau de scolarisation permet de rapprocher les non-bénéficiaires des bénéficiaires sur le plan des contributions et des rétributions. Le programme apparaît donc plus efficace pour les nonscolarisées que pour les plus scolarisées. Cela voudrait dire que le programme comble chez les non-scolarisées ce que l'éducation donne aux scolarisées.

\section{DISCUSSION SUR LA PORTÉE DES RÉSULTATS POUR L'INSERTION DES FEMMES DANS LE DÉVELOPPEMENT}

Cette étude permet d'avancer qu'une action comme celle réalisée par le programme CEC aide les femmes pauvres à faire face à leur situation et à s'intégrer dans le développement économique et social. Dans une perspective de contribution de l'action sociale à l'insertion des femmes pauvres dans le développement, l'analyse fait ressortir les points saillants suivants : la contribution des femmes, les expériences économiques et le crédit, l'éducation et la participation politique.

\section{La contribution des femmes}

L'analyse des résultats sur l'utilisation des revenus a révélé une logique de contribution des femmes. C'est un apport appréciable et multiforme. Les répondantes investissent une bonne quantité de leurs gains dans la consommation de la famille. Nous ne possédons pas plus d'information dans ce domaine, puisque notre recherche s'intéresse au milieu extrafamilial. Nous retenons toutefois que l'utilisation des bénéfices pour la consommation est liée au rôle de la femme qui est en charge de l'entretien du foyer, surtout dans un contexte où ses responsabilités augmentent.

Les bénéficiaires du programme CEC investissent aussi dans l'épargne et l'agrandissement de l'activité économique. L'épargne est souvent investie dans la tontine avec des destinations diverses. On distingue par exemple les tontines de toit chez les femmes léboues du Sénégal, les tontines de vaisselle, de meubles, de cérémonies comme le baptême, le deuil (Henry et collab., 
1991). Cet apport économique, qui est une nouvelle forme de contribution, pourrait aussi être un investissement dans une éventuelle promotion sociale pour changer les rapports de pouvoir dans la famille.

La contribution dans le milieu est importante; c'est une participation sociale surtout sur le plan culturel (mariage, baptême, décès, initiation). Même si la CEC ne finance pas spécifiquement les cérémonies, la souplesse et la flexibilité de son organisation donnent aux femmes beaucoup de latitude. Elles évitent l'endettement et élaborent d'autres stratégies d'épargne pour faire face à leurs obligations sociales, les "Nat » ou tontines de cérémonie (Henry et collab., 1991). Les cérémonies représentent un moyen d'expression dans le milieu. Elles renforcent les liens de solidarité, surtout en milieu urbain, et sont aussi une forme d'assurance pour un soutien mutuel qui allège le poids de la cérémonie. La CEC donne aux femmes les moyens d'avoir des revenus pour une participation de qualité dans leur milieu; c'est un « marketing social » qui renforce la position dans le milieu et rehausse le statut social. L'importance de la participation sociale s'explique par l'interrelation des rôles sur les plans culturel, social, économique qui font la spécificité des femmes.

\section{Les expériences économiques et le crédit}

Les femmes du programme de la CEC font partie des couches défavorisées de la population des zones périurbaines de Thiès qui vivent une marginalisation économique et sociale liée aux rapports sociaux entre les sexes. Sans éducation ni formation, elles n'ont pas accès à l'emploi et elles sont pauvres. Le programme a considéré ces facteurs pour s'adapter aux besoins des bénéficiaires.

Des expériences de femmes qui ont réussi, comme celles de la Grameenbank et de la banque de Madras, sont parties d'une analyse de genre qui intègre les facteurs structurels (culturel, social, politique, économique) et de l'analyse de la situation des femmes dans un contexte global (Rathgeber, dans Dagenais et Piché, 1994). Les expériences qui n'intègrent pas la dimension femme sous l'angle de la spécificité des rôles féminins dans la société sont des échecs. Elles sont récupérées par d'autres acteurs dans le milieu (Rossi, 1993), ou sont abandonnées par les 
femmes faute de retombées capables de soutenir une promotion durable.

L'accès au crédit permet de percer la réalité du cercle vicieux des femmes «qui sont pauvres parce qu'elles n'ont pas de crédit, et n'ont pas accès au crédit parce qu'elles sont pauvres " (Camiré, dans Champagne, 1989). L'importance du crédit pour les femmes pauvres apparaît dans les conclusions d'une étude sur les femmes du Sahel. Parmi les intrants susceptibles d'augmenter la productivité des femmes autres que le travail se trouve le capital sous forme de crédit (Dixon-Mueller et Anker, 1989). L'utilisation du savoir-faire local comme la CEC est organisée à l'image de la tontine. Elle permet une pleine participation, confirmant cette pensée : "Les femmes pauvres comprennent mieux que quiconque leur situation et détiennent des solutions qu'il suffit de soutenir » (Labrecque, 1988). La CEC est entièrement dirigée par le comité de gestion des bénéficiaires. Elle présente des similitudes avec l'expérience de la Grameenbank du Bangladesh pour ce qui est de la responsabilisation des utilisatrices, qui contrôlent le conseil d'administration (Hook, 1990). Ainsi qu'il est constaté dans la théorie, les femmes pauvres ont les mêmes contraintes et les mêmes besoins; l'adaptation du crédit à leur spécificité est un gage de succès.

\section{L'éducation et la participation politique}

Le taux d'analphabétisme dans la population féminine adulte est de l'ordre de $82 \%$ au Sénégal (EDS II, 1994). Cette situation est liée aux préjugés et stéréotypes à l'égard des femmes qui les ont éloignées de toute formation scolaire ou qualification professionnelle. Par conséquent, les femmes ne sont pas compétitives pour l'emploi et sont absentes des instances de décision (Diop, 1993 ; Sow, 1995a). L'éducation est donc l'un de leurs besoins prioritaires (Nations Unies, 1987). Le programme CEC se présente comme un programme de formation sur le terrain, un apprentissage sur le tas. Mais le rôle déterminant de la scolarité chez les nonbénéficiaires montre l'importance de l'éducation. Comme il a déjà été constaté dans la théorie, l'éducation serait le meilleur moyen de lutter contre la pauvreté des femmes. Lorsqu'on regarde le cas du programme CEC, on constate que les femmes éduquées, même lorsqu'elles ne participent pas à un programme, sont inté- 
grées sur le plan social. En attendant d'avoir une généralisation de l'éducation auprès des femmes, les programmes comme la CEC jouent un rôle important dans la lutte contre la pauvreté.

Le programme CEC ne débouche pas sur une participation politique des femmes. Les bénéficiaires n'exercent pas leur rôle public plus que les autres femmes. II faudrait les aider à se mobiliser sur le plan politique et à incorporer cet aspect dans les futures actions du CEC.

\section{CONCLUSION}

Les résultats de cette recherche, malgré les limites de celle-ci, peuvent favoriser une clarification des composantes de l'intégration socio-économique des femmes pauvres en milieu urbain et périurbain. En même temps, ils peuvent servir à améliorer l'action à l'intérieur du programme. La recherche a une utilité pour la pratique par l'accent qui y est mis sur les aspects alphabétisation, formation en gestion, promotion de la scolarisation des filles par l'intermédiaire de l'Information Éducation et Communication (IEC) ainsi que par l'analyse de genre, qui intègre aussi dès le début des aspects sociopolitiques.

Cette recherche est une première dans l'évaluation scientifique des programmes sociaux à l'intérieur de la Direction de l'action sociale au Sénégal. Ses résultats pourront guider des actions dans le domaine du crédit et servir de point de départ pour des études plus approfondies. En ce qui concerne les décideurs, ils pourront leur servir de base dans la perspective de restructuration et de régionalisation des services sociaux. Les régions vont vers une plus grande autonomie pour une meilleure prise en compte de leur spécificité. Les projets d'autopromotion de cette nature pourraient contribuer à rendre le service social plus pertinent et plus performant auprès des usagères.

Maïmouna SOURANG

Directrice régionale

Centre de protection et de réinsertion sociale, Thiès, Sénégal Conseil experte auprès de la ministre de la Femme et de la Famille Dakar, Sénégal 


\section{André BEAUDOIN \\ Professeur titulaire \\ École de service social, Université Laval \\ Élaine CAREY-BÉLANGER \\ Professeure titulaire \\ École de service social, Université Laval}

\section{Références bibliographiques}

AFARD (1994). Association des femmes africaines pour la recherche et le développement. Femmes et démocratisation en Afrique. Document du Forum des ONG de la Pré-conférence mondiale des Nations Unies sur les femmes, Beijing, 13-15 novembre, Dakar, Sénégal.

AUBIN, B. (1991). "Aménagement des terroirs et participation populaire ", Élément du débat sur les approches participatives, Québec, Université Laval, série Dossiers $\mathrm{n}^{\circ} 15,84 \mathrm{p}$.

AUDIBERT, A. (1977). "Le service social en Afrique francophone dans une perspective de développement, l'Afrique coloniale. » Tomes I et II, Paris, Thèse de doctorat de spécialité en Sciences sociales du travail.

BEAUDOIN, A. (1987). "Le champ des services sociaux dans la politique sociale au Québec. " Programme de recherche, recueil de résumés. Québec, Commission d'enquête sur les services de santé et les services sociaux.

BIRou, A. (1986). Vocabulaire pratique des sciences sociales, Paris, Éditions ouvrières.

BISILLIAT, J. et M. LECARME (1992). «Territoires du féminin, territoires du masculin. Relation de genre et développement », Femmes et Sociétés, Paris, ORSTOM Éditions.

BOSERUP, E. et C. LILJENCRANTZ (1975). Intégration de la femme au développement, Pourquoi, Quand, Comment? Programme des Nations Unies pour le développement (PNUD).

Boserup, E. (1983). La femme face au développement économique. Traduit de l'anglais par Marie C. Marache, Paris, Presses universitaires de France.

Boudon, R., P. Besnard, M. Cherkaoul et B.-P. LÉCuyer (dir.) (1989). Dictionnaire de la sociologie, Paris, Larousse. 
CAREY-BÉlANGer, É. et M. SourANG (1994). "Vingt groupements et associations de femmes de la commune de Thiès. " Québec, Composante Femmes-Société-Développement, Centre Sahel, Université Laval.

CEA (1994). Commission économique des Nations Unies pour l'Afrique. Document de la $5^{\mathrm{e}}$ Conférence régionale africaine sur les femmes. Dakar, Sénégal.

Champagne, S. (1989). Les femmes et le développement. Stratégies, moyens, impact. Des coopérantes témoignent de leurs expériences avec les femmes du Sahel. Textes colligés. Québec, Centre Sahel, Université Laval.

Dagenais, H. et D. PICHÉ (1994). Femmes, Féminisme et Développement, Montréal, McGill-Queen's University Press.

Didier, M. (1984). Économie. Les règles du jeu, Paris, Éditions Economica.

DıOP, M. (1993). Participation sociale et économique et intégration dans le milieu de vie des participantes du Foyer Clair-logis, Thiès, Sénégal. Québec, Mémoire de maîtrise en service social, Université Laval.

DIXON-MuelleR, R. et R. ANKER (1989). Évaluation de la contribution des femmes au développement économique, Genève, Bureau international du travail (BIT).

Dumouchel, S. et N. THĖde (1986). Femmes, épargne et crédit au Sahel, Montréal, projet Solidarité Canada-Sahel.

DUTRÉNIT, J.-M. (1989). Gestion et évaluation des services sociaux, Paris, Éditions Economica.

EDS II (1994). Enquête démographique et de santé au Sénégal, Dakar, Division des statistiques démographiques.

FERRÉOL, G. (1991). Dictionnaire de sociologie, Paris, Armand Colin Éditeur. (Coll. Cursus)

GraWITZ, M. (1988). Lexique des sciences sociales, Paris, Dalloz.

HenRY, A., G.H. TCHeNTE et PH.G. Dieumegard (1991). Tontines et banques au Cameroun, les principes de la société des amis, Paris, Karthala.

Hook, B. (1990). L'accès et l'utilisation du crédit par les femmes au Rwanda. Mémoire de maîtrise d'économie rurale, Québec, Université Laval.

INSTITUT INTERNATIONAL DE RECHERCHE ET DE FORMATION DES NATIONS UNIES POUR LA PROMOTION DE LA FEMME (INSTRAW) (1987). Rapport sur la promotion des femmes, Harare, Zimbabwe, Commission économique pour l'Afrique (CEA). 
KANOUTE, M. (1990). Thiès: une région, des potentialités, une stratégie de planification. Diplôme de $3^{\mathrm{e}}$ cycle en urbanisme, Dakar, Sénégal.

LABRECQUE, M.-F. (1988). «Femmes, développement, idéologie, cas du Mexique », Recherches féministes, vol. 1, n ${ }^{\circ}$ 2, p. 53-68.

MiANDA, G. (1996). Femmes africaines et pouvoir. Les maraîchères de Kinshasa, Paris, L'Harmattan. (Coll. Zaïre-Histoire et Société)

NATIONS Unies (1987). Politique de participation de la population, moyen de faire progresser l'intégration sociale, New York, Nations Unies.

Rossı, C. (1993). Évaluation des enjeux sociaux : cas d'une coopérative d'épargne et de crédit au Mali, Québec, Publications du Centre Sahel, Université Laval.

SARR, F. (1991). Marginalité urbaine et développement, une contradiction autogérée par les femmes, Paris. Thèse pour le nouveau doctorat en Anthropologie et Sociologie politique, Université Paris VI.

Savane, M.-A. (dir.) (1986). Femmes et développement en Afrique de l'Ouest: Incidences des transformations socio-économiques sur le rôle et le statut des femmes, Genève, Institut de recherche des Nations Unies pour le développement social (UNRISD).

SILVA, É. et L. JUTEAU (1993). "Contribution à l'amélioration de la position sociale des femmes sénégalaises», dans Femmes sénégalaises: Réalités et enjeux stratégiques, Dakar, Sénégal, Centre canadien d'étude et de coopération internationale (CECI).

Sow, F. (1995a). "L'analyse de genre et les sciences sociales en Afrique », dans Imam Ayesha, Amina Mawa et Fatou Sow (dir.), Engineering Africa Social Sciences, Dakar, Sénégal, CODESRIA.

Sow, F. (1995b). Femmes rurales chefs de famille en Afrique subsaharienne, sous l'égide du Service pour l'intégration des femmes au développement, Rome, Italie, Food and Agriculture Organization (FAO).

TINKER, I. (1979). "Le développement contre les femmes », Questions féministes, 6, p. 69-86.

VoIX DU SUD (1992). Femmes du sud, Autres voix pour le $X X^{e}$ siècle, traduit de l'anglais par Alice Hodgson et Dawn P. Lanaud, Paris, Côté-Femmes Éditions.

Wilensky, H. et C. Lebeaux (1965). Industrial Society and Social Welfare, New York, Free Press. 